\title{
Correction to: Oncology practitioners' perspectives and practice patterns of post- treatment cancer survivorship care in the Asia-Pacific region: results from the STEP study
}

Raymond Javan Chan ${ }^{1,2^{*}}$, Patsy Yates ${ }^{1,2}$, Qiuping Li ${ }^{3}$, Hiroko Komatsu ${ }^{4}$, Violeta Lopez ${ }^{5}$, Myat Thandar ${ }^{6}$, Selva Titus Chacko ${ }^{7}$, Winnie Kwok Wei So ${ }^{8}$, Kanaungnit Pongthavornkamol ${ }^{9}$, Myungsun $\mathrm{Yi}^{10}$, Pongpak Pittayapan ${ }^{11}$, Jesson Butcon ${ }^{12}$, David Wyld ${ }^{2}$, Alex Molassiotis ${ }^{13}$, on behalf of the STEP study collaborators

\section{Correction}

It has been highlighted that the original manuscript [1] contains a typesetting error in the name of Jesson Butcon. This was incorrectly captured as Jessica Butcon in the original manuscript which has since been updated.

\footnotetext{
Author details

'School of Nursing and Institute of Health and Biomedical Innovation, Queensland University of Technology, Brisbane, Australia.

${ }^{2}$ Cancer Care Services, Royal Brisbane and Women's Hospital, Brisbane, Australia.

${ }^{3}$ Wuxi Medical School, Jiangnan University, Wuxi, Jiangsu, China.

${ }^{4}$ Faculty of Nursing and Medical Care, Keio University, Tokyo, Japan

${ }^{5}$ Alice Lee Centre for Nursing Studies, Yong Loo Lin School of Medicine, National University of Singapore, Singapore, Singapore.

${ }^{6}$ The University of Nursing, Yangon, Myanmar.

${ }^{7}$ College of Nursing, Christian Medical College, Vellore, India.

${ }^{8}$ The Nethersole School of Nursing, The Chinese University of Hong Kong, Hong Kong, China.

${ }^{9}$ Faculty of Nursing, Mahidol University, Bangkok, Thailand.

${ }^{10} \mathrm{College}$ of Nursing and Research Institute of Nursing Science, Seoul National University, Seoul, Republic of Korea.

${ }^{11}$ Nursing Department of Siriraj Hospital, Mahidol University, Bangkok, Thailand.

${ }^{12}$ College of Medicine, Bicol University, Bicol, Philippines.

${ }^{13}$ School of Nursing, Hong Kong Polytechnic University, Hong Kong, China.
}

Received: 8 February 2018 Accepted: 8 February 2018

Published online: 01 March 2018

\section{Reference}

1. Chan, et al. Oncology practitioners' perspectives and practice patterns of post-treatment cancer survivorship care in the Asia-Pacific region: results from the STEP study. BMC Cancer. 2017;17:715. https://doi.org/10.1186/ s12885-017-3733-3.

\footnotetext{
* Correspondence: raymond.chan@qut.edu.au

${ }^{1}$ School of Nursing and Institute of Health and Biomedical Innovation Queensland University of Technology, Brisbane, Australia

${ }^{2}$ Cancer Care Services, Royal Brisbane and Women's Hospital, Brisbane, Australia
} 